\title{
Application of Parallel-hierarchical Transformations for Rapid Recognition of Dynamic Images Based on GPU Technology
}

\author{
Leonid Timchenko \\ State University for Transport \\ Economy and Technologies \\ SUTET \\ Kyiv, Ukraine \\ timchen@list.ru
}

\author{
Andriy Yarovyy \\ Vinnytsia National Technical \\ University \\ VNTU \\ Vinnytsia, Ukraine \\ axa@vinnitsa.com
}

\author{
Nataliya Kokriatskaia \\ State University for Transport \\ Economy and Technologies \\ SUTET \\ Kyiv, Ukraine \\ kokriatskaia@rambler.ru
}

\author{
Igor Ivasyuk \\ State University for Transport Economy and \\ Technologies \\ SUTET \\ Kyiv, Ukraine \\ tihor_vin@ukr.net
}

\author{
Alyona Denysova \\ State University for Transport Economy and \\ Technologies \\ SUTET \\ Kyiv, Ukraine \\ fate2107@yandex.ru
}

\begin{abstract}
The paper presents a method of parallel-hierarchical transformations for rapid recognition of dynamic images using GPU technology. Direct parallel-hierarchical transformations based on cluster CPU-and GPU-oriented hardware platform. Mathematic models of training of the parallel hierarchical (PH) network for the transformation are developed, as well as a training method of the PH network for recognition of dynamic images. This research is most topical for problems on organizing high-performance computations of super large arrays of information designed to implement multi-stage sensing and processing as well as compaction and recognition of data in the informational structures and computer devices. This method has such advantages as high performance through the use of recent advances in parallelization, possibility to work with images of ultra dimension, ease of scaling in case of changing the number of nodes in the cluster, auto scan of local network to detect compute nodes.
\end{abstract}

Keywords- parallel-hierarchical (PH) network, laser beam spot images, parallel-hierarchical (PH) transformation, fast recognition of dynamic images, GPU technology.

\section{INTRODUCTION}

Rapidly growing requirements to modern computational media encourage development of new intelligent methods of information transfer and processing. This problem can be solved through application of the laser-based technologies [1-3]. One of the most efficient methods of large data arrays processing is their parallel processing based on the neurallike parallel hierarchical systems. A goal of this paper is to develop and apply the suggested method and means of parallel hierarchical transformation for fast recognition of dynamic images. To increase a speed of computational structures, the method based on the normalizing equation was applied. It also allows performing a procedure of determination of weighting coefficients for each class [4].

\section{THE METHOD OF PARALLEL HIERARCHICAL} TRANSFORMATION WITH FORMATION OF THE NORMALIZING EQUATION FOR FAST EQUATION FOR FAST IMAGE RECOGNITION

Algorithm and software package [5-7] realize the method of parallel hierarchical transformation with formation of the normalizing equation for fast image recognition, which is described in details in the previous works.

The research based on a masking method of information processing for purposes of fast recognition of laser beam spot images [8] suggests the need to improve a method of the parallel hierarchical transformation.

A number of hidden layer elements can be determined from the length of the network algorithm, accordingly formalizing a procedure for calculation of the number of hidden layer elements. Averaged values of weighting coefficients are determined by (1):

$$
\bar{w}_{t}=\frac{\sum_{p=1}^{N} w_{t}^{(p)}}{N}, t=\overline{1, k-1}
$$

where $N$ is a dimensionality of the taught sample $P$.

Let us compile a system of equations to determine tuning coefficients $w_{1} \div w_{k-1}$ as system (2): 


$$
\begin{aligned}
& w_{1}=\frac{\sum_{t=2}^{k} a_{11}^{t}}{\left(a_{11}^{2}+\sum_{i} a_{i}^{2}\right)} \\
& w_{2}=\frac{\sum_{t=2}^{k} a_{11}^{t}}{\left(a_{11}^{3}+\sum_{i} a_{i}^{3}\right)}-\frac{w_{1} a_{11}^{2}}{\left(a_{11}^{3}+\sum_{i} a_{i}^{3}\right)} \text {, } \\
& w_{k-2}=\frac{\sum_{t=2}^{k} a_{11}^{t}}{\left(a_{11}^{k-1}+\sum_{i} a_{i}^{k-1}\right)}-\frac{w_{1} a_{11}^{2}+w_{2} a_{11}^{3}+\cdots+w_{k-3} a_{11}^{k-2}}{\left(a_{11}^{k-1}+\sum_{i} a_{i}^{k-1}\right)} \text {, } \\
& w_{k-1}=\frac{\sum_{t=2}^{k} a_{11}^{t}}{\left(a_{11}^{k}+\sum a_{i}^{k}\right)}-\frac{w_{1} a_{11}^{2}+w_{2} a_{11}^{3}+\cdots+w_{k-2} a_{11}^{k-1}}{\left(a_{11}^{k}+\sum a_{i}^{k}\right)},
\end{aligned}
$$

Having compared the efficiency of the parallel hierarchical transformation [9-13] and known transformations by a number of computational operations used, a conclusion can be made that a number of operations for the $\mathrm{PH}$ transformation is $N(N+1)$, where $N$ is a total number of elements processed.

\section{EXPERIMENTAL RESULTS AND PHYSICAL MODELING OF THE PARALlEL HIERARCHICAL TRANSFORMATION FOR \\ FAST RECOGNITION OF IMAGES OF LASER BEAM SPOTS}

Four routes were used in experimental research of the parallel hierarchical transformation for fast recognition of images of laser beam spots [14-17]. Each route contains 100 frames. Using the normalizing equation (2), let us determine normalized measures for route 1 (Fig. 1 ).

An average percentage of "good" images in this case is $40 \%$ and $59 \%$ respectively. Then the $\mathrm{PH}$ network is trained for repeated processing of "bad" route fragments [18].

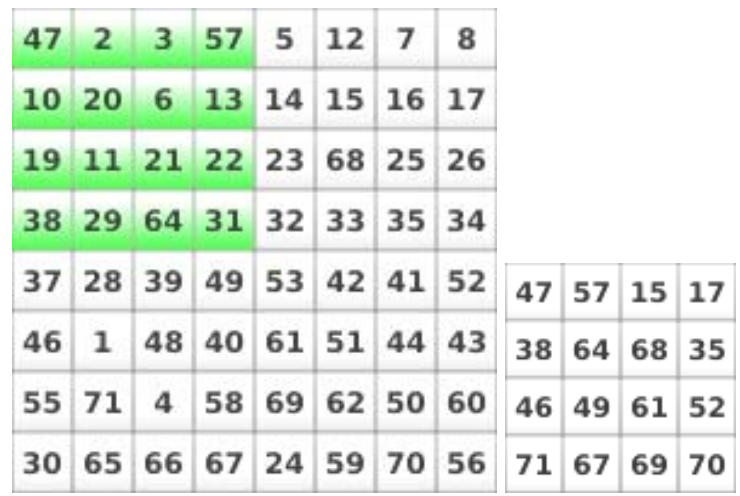

a)

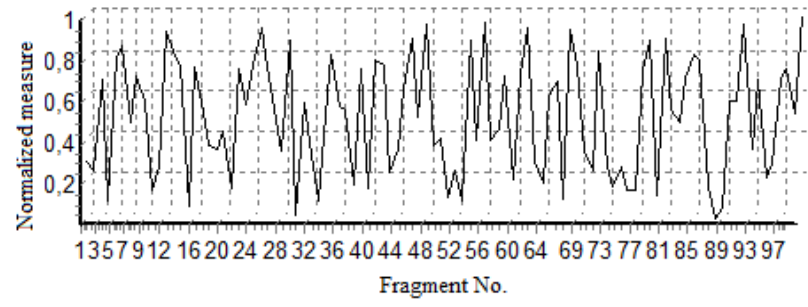

Figure 1. Normalized measures for route 1

Image processing is based on the GPU with cores realized at all elements covered by the initial region. The only obvious way to calculate a scalar of the input vector is a representation of $1 \times 1$ initial elements and use of the core read in all values from the input texture. This approach has several drawbacks [19, 20]. First, only one of parallel elementary processors would be busy. Second, that would probably exceed a maximum permitted by shader length and static instruction of calculation for some hardware. That is why we will perform a parallel operation of reduction based on global methods of communication on parallel computers.

At the high level, GPU-based parallel calculation is a correction of sizes of the input and output texture and index elements. For each of its elements, coordinates for input texture are corrected in such a way that they correspond to disconnected $2 \times 2$ subregions. Then values in those subregions are compared. This is repeated recursively until the $2 \times 2$ texture is reduced to the final one by the $1 \times 1$ "scalar" texture through a logarithmic series of repetitions [21-23].

Next series of images finalize the first step of reduction of the $8 \times 8$ algorithm of the input texture (Fig. 6). The left image demonstrates the input texture. Initial elements are marked in green (Fig. 2a). The right image is a result of the first round of reduction. Each initial element contains a local maximum of transfer of the $2 \times 2$ subregion in the input texture. This relation, in addition, is distinguished in the second line of images (Fig. 2b).

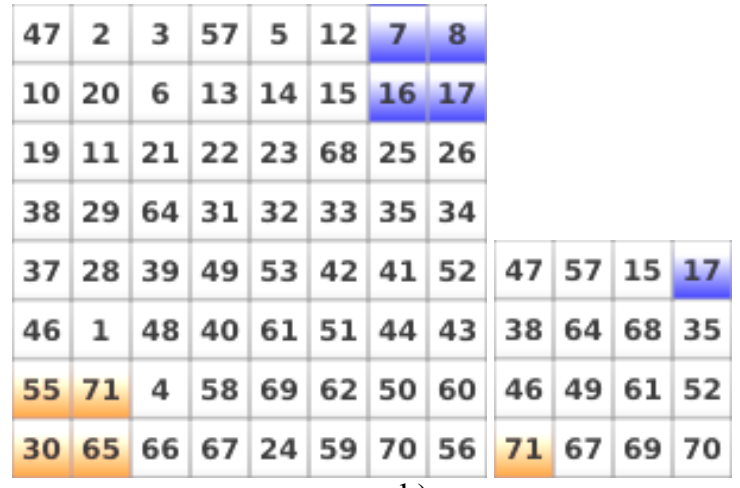

b)

Figure 2. The first step of reduction of the $8 \times 8$ algorithm of the input texture 
Let us determine coordinates of energy centers of the fragment of route 1 (Fig. 3):

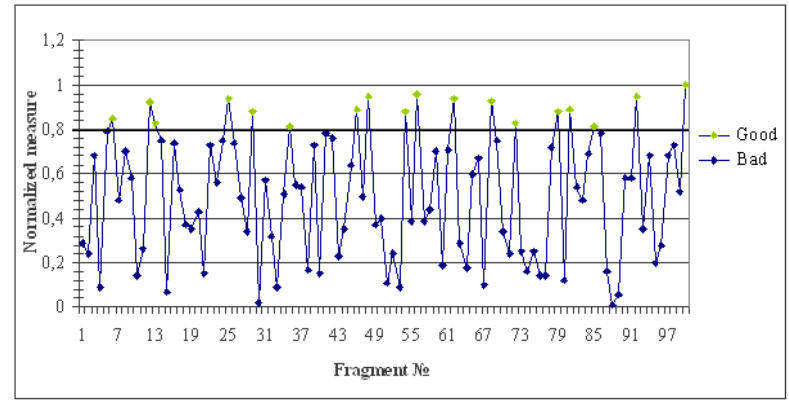

Figure 3. Graph of "good" and "bad" images formation in the track number 1.

A graphical interpretation of the distribution of coordinates of energy centers is shown in Fig. 4.

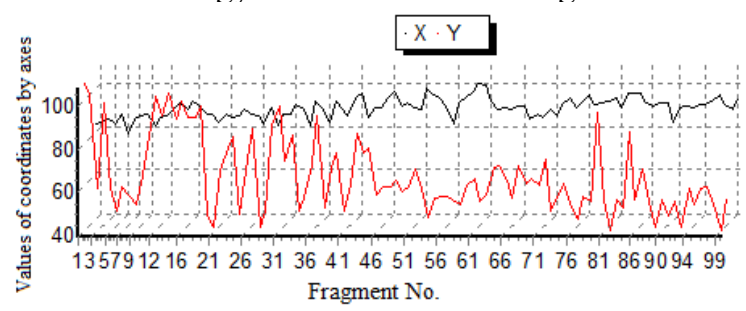

Figure 4. A graphic interpretation of the distribution of coordinates of energy centers

After the training of the PH network, a portion of "good" images was $83 \%$ (as compared to $18 \%$ ).

The graphical interpretation of the determination of energy center coordinates after the $\mathrm{PH}$ network training is demonstrated in Fig. 5.

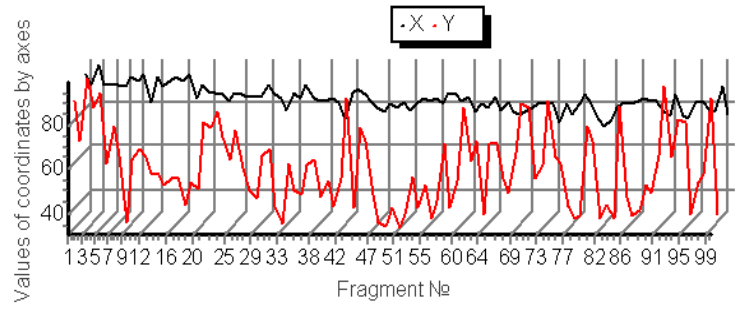

Figure 5. Determination of the energy centers coordinates for the fragments of route number 2

After training of the PH network percentage of "good" images on track number 2 was $76 \%$ (12\% before training), Fig. 6.

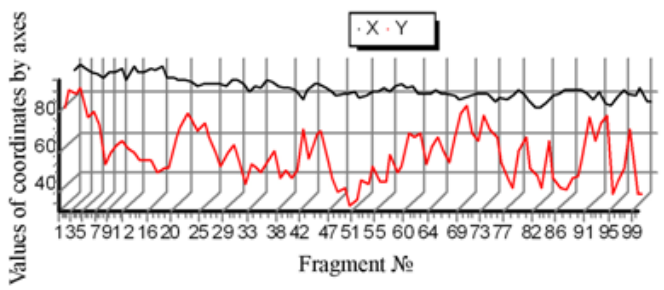

Figure 6. Determination of the energy centers coordinates for the fragments of route number 2 after $\mathrm{PH}$ network training

The graphical interpretation of the determination of energy center coordinates for route number 3 is demonstrated in Fig. 7.

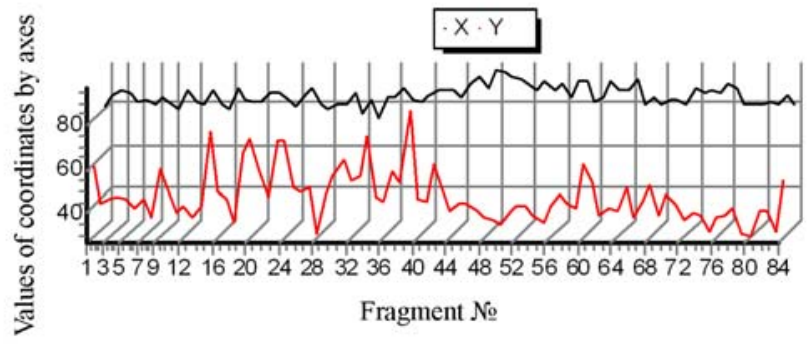

Figure 7. Determination of the energy centers coordinates for the fragments of route number 3

After training of the PH network percentage of "good" images on track number 2 was $65 \%$ (15\% before training), Fig. 8.

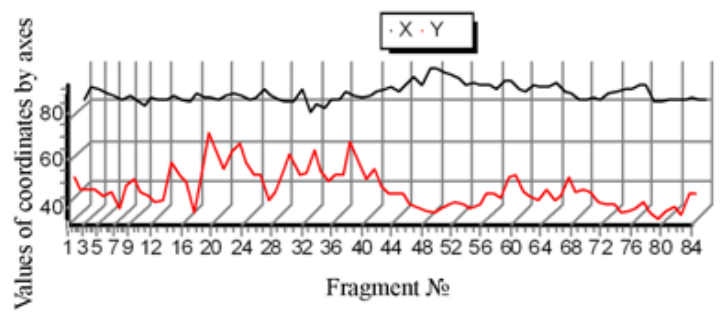

Figure 8. Determination of the energy centers coordinates for the fragments of route number 3 after $\mathrm{PH}$ network training

After training of the PH network percentage of "good" images on track number 2 was $83 \%$ (17\% before training), Fig. 9.

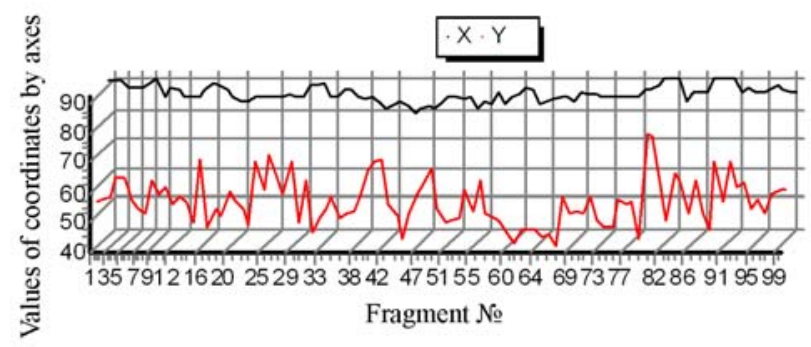

Figure 9. Determination of the energy centers coordinates for the fragments of route number 4 after $\mathrm{PH}$ network training

\section{CONCLUSIONS}

The paper deals with a topical problem of increasing efficiency of recognition of dynamic images. The analysis of development of the PH transformation allows to attribute the suggested parallel hierarchical approach to neural methods of 
transformation with a network of direct recognition and the space-time organization of connections. A method of the PH transformation with formation of the normalizing equation is developed. A method of $\mathrm{PH}$ transformation containing measures of tuning coefficients correspondence of the reference PH network and current network, was developed, as well as with finding a measure of correspondence of two networks as a whole.

Algorithms of processing and recognition of images of laser beam spots were developed. The developed algorithms allow determining a position of energy centers, classifying frames of images of the laser route.

On the basis of the developed algorithms, software was created for modeling a neural-like $\mathrm{PH}$ network. This software is used for classification and fast processing of images.

The method of $\mathrm{PH}$ transformations for rapid recognition of dynamic images using GPU technology has such advantages as high performance through the use of recent advances in parallelization, possibility to work with images of ultra dimension, ease of scaling in case of changing the number of nodes in the cluster, auto scan of local network to detect compute nodes [24, 25]. Decreasing of time required for pre-processing and network processing of laser routes image fragments should also be noted.

In [14-17] analysis of neural network technology using for extended laser route image classification was held. Computer simulation showed that the percentage of correctly recognized images was $92.5 \%$, moreover $74 \%$ of "good" images and $60 \%$ of "bad" ones. In [15] simulation of recognition system based on neural network MLP and neural network based on radial basis function (RBF) was conducted. During recognition the sample of 140 spots-images of laser route by neural network based on RBF, modeled in the package Statistica Neural Networks 4.0, 92\% of correctly recognized images received. Comparing the results of $[14,15]$ and the results of this paper we can talk about the availability of the last one.

\section{REFERENCES}

[1] J. M. Khosrofian and B. A. Garetz, Measurement of a Gaussian laser beam diameter through the direct inversion of knife-edge data, J. Appl. Opt. 22 (1983) 3406-3410.

[2] N.G. Basov, E.M. Zemskov, Y.F. Kutaev, V. M. Kazansky, G. A. Kirillov, S. M. Kulikov, O. Y. Nosach, S. A. Sukharev, Laser Control of Near Earth Space and Possbilities for Removal of Space Debris from Orbit with Explosive Photo-Dissociation Lasers with Phase Conjugation, Proc. GCL/HPL 98, SPIE Symposium, StPetersburg (Russia), vol. 3574, 219-228 (1998).

[3] L. I. Timchenko, Y. F Kutaev, V. P. Kozhemyako, Method for Training of a Parallel-Hierarchical Network, Based on Population Coding for Processing of Extended Laser Paths Images, Proceedings of SPIE, vol. 4790, 465-479 (2002).

[4] Timchenko L. I., Kokryatskaya N. I., Melnikov V.A method of organization of a parallel-hierarchical network for image recognition, J. Cybernetics and systems analisis, 47(1) (2011) 140151 .

[5] Yarovyy A.A. Image processing by direct parallel-hierarchical transformation based on cluster systems with distributed information processing, J. Visnyk of Vinnitsya Technical Institute, No 3 (2011), pp.133-140..

[6] D.M. Chitty, A data parallel approach to genetic programming using programmable graphics hardware, GECCO '07: Proceedings of the $9^{\text {th }}$ annual conference on genetic and evolutionary computation, vol. 2, pp. 1566-1573, Jul., (1991).

[7] Z. Luo, H. Liu and X. Wu, Artificial neural network computation on graphic process unit, IJCNN '05. Proceedings of the IEEE International Joint Conference on Neural Networks, vol. 1, pp. 622-626, Jul, (2005).

[8] Timchenko L. I., Kokryatskaya N. I., Melnikov V.A method of organization of a parallel-hierarchical network for image recognition, J. Cybernetics and systems analisis, 47(1) (2011) 140151.

[9] J.M. Li, D.L. Wan, Z.X. Chi and X.P. Hu, 2006, A parallel particle swarm optimization algorithm based on fine-grained model with GPUaccelerating, Harbin Gongye Daxue Xuebao/Journal of Harbin Institute of Technology, vol. 38, no. 12, pp. 2162-2166, Dec.,

[10] R. Xu and D.C. Wunsch II, Clustering. IEEE / Wiley Press, Hoboken, NJ, (2008).

[11] D. Everitt, S. Landau and M. Leese, Clustering analysis, 4th edition. Arnold, London, UK, (2001).

[12] D.C. Wunsch II, ART properties of interest in engineering applications, Proc. IEEE/INNS International Joint Conference on Neural Networks, Atlanta, GA, (2009).

[13] D. Knuth, The Art of Computing Programming: Fundamental Algorithms, 3rd Edition, vol. 1, Addison-Wesley, (1997).

[14] Sung Uk Jung, Yun Su Chung, Jang Hee Yoo, Kyo II Chung Patent № US007697734B2 Method and apparatus of detecting eye using symmetry and moment characteristics of object, Apr, 13, (2010).

[15] Sung Uk Jung, Yun Su Chung, Jang Hee Yoo, Kyo II Chung Patent № US007697734B2 Method and apparatus of detecting eye using symmetry and moment characteristics of object, Apr, 13, (2010).

[16] Simon Xinmeng Liao Image Analysis by Moments A Thesis Submitted to the Faculty of Graduate Studies in Partial Fulfillment of the Requirements for the Degree of Doctor of Philosophy the University of Manitoba Winnipeg, Manitoba, Canada, 1993.

[17] R.J.Prokop and A.P.Reeves, A Survey of Moment-Based Techniques for Un occluded Object Representation and Recognition, J. Graphical Models And Image Processing, Vol. 54, №5, (1992) 438-460.

[18] C.H.Teh and R.T. Chin, , On image analysis by the methods of moments, J. IEEE Trans. Pattern Anal. Machine Intell. Vol. PAMI10 (1988) 496-512.

[19] Sejun Kim, A GPU based Parallel Hierarchical Fuzzy ART Clustering, Advanced Res, (2003).

[20] R.J.Prokop and A.P.Reeves, A Survey of Moment-Based Techniques for Un occluded Object Representation and Recognition, J. Graphical Models And Image Processing, Vol. 54, №5, (1992) 438-460.

[21] Simon Xinmeng Liao Image Analysis by Moments A Thesis Submitted to the Faculty of Graduate Studies in Partial Fulfillment of the Requirements for the Degree of Doctor of Philosophy the University of Manitoba Winnipeg, Manitoba, Canada, 1993.

[22] Sung Uk Jung, Yun Su Chung, Jang Hee Yoo, Kyo II Chung Patent № US007697734B2 Method and apparatus of detecting eye using symmetry and moment characteristics of object, Apr, 13, (2010).

[23] A.J. Martin, S.M. Burns, T.K. Lee, D.Borkovic, and P.J. Hazewindus, "The design of an asynchronous microprocessor," Advanced Res. VLSI: Proc. Decennial Caltech Conf. VLSI: Proc. Decennial Caltech Conf. VLSI, MIT Press Cambridge, MA, Mar., (1986). 
[24] M. Gorchetchnikov, H. Ames, M. Versace, Simulating Biologically Realistic Neural Models on Graphics Process Units, ICCNS 2008, Boston, MA, (2008).

[25] M. Martnez-Zarzuela, F. Pernas, A. de Pablos, M. Rodrguez, J. Higuera, D. Giralda and D. Ortega, Adaptative Resonance Theory
Fuzzy Networks Parallel Computation Using CUDA, Bio-Inspired Systems: Computational and Ambient Intelligence, vol. 5517, pp. 149-156, (2009). 